\title{
EVALUATION PROTOCOL FOR THE WIND SYSTEM ATMOSPHERIC MODELS (U)
}

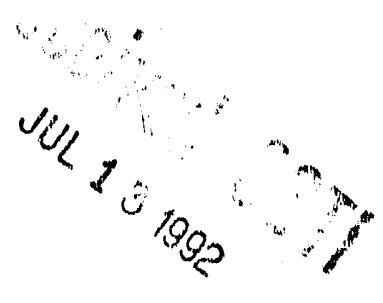

by

\author{
J. D. Fast
}

Westinghouse Savannah Rive: Company

Savannah River Laboratory

Aiken, South Carolina 29808

A DP Report

This paper was prepared in connection with work done under Contract No. DE-ACO9-89SR18035 with the U.S. Department of Energy. By acceptance of this paper, the publisher and/or recipient acknowledges the U.S. Govermment's right to retain a nonexclusive, royalty-free license in and to any copyright covering this paper. along with the right to reproduce and to authorize others to reproduce all or part of the copyrighted paper.

\section{DISTRIBUTION OF THIS DOCUMENT IS UNLIMTTED \\ DISCLAIMER

\footnotetext{
This repont was prepared as an account of work sponsored by an agency of the United States Government. Neither the United States Government nor any agency thereof, nor any of their employees, makes any warranty, express or implied, or assumes any legal liability ot responai. bility for the accuracy, completeness, or usefulness of any information, apparatus, product, or process disclosed, or represents that its use would not infririge privately owned rights. Refer. ence herein to any specific commercial product, process, or service by trade name, trademark, manufacturer, or otherwise does not necessarily constitute or imply its endorsement, recom. mendation, or favoring by the United States Government or any agency thereof. The views

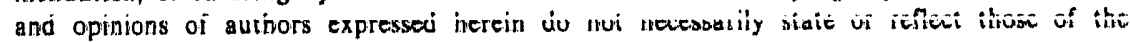
United States Government or any agency thereof.
} 


\title{
EVALUATION PROTOCOL FOR THE WIND SYSTEM ATMOSPHERIC MODELS
}

\author{
By J. D. Fast \\ Westinghouse Savannah River Company \\ Savannah River Site \\ Aiken, SC 29808
}

\subsection{INTRODUCTION}

Atmospheric transport and diffusion models have been developed for real-time calculations of the location and concentration of toxic or radioactive materials during an accidental release at the Savannah River Site (SRS). These models have been incorporated into an automated menu-driven computer based system called the WIND (Weather INformation and Display) system.

In an effort to establish more formal quality assurance procedures for the WIND system atmospheric codes, a software evaluation protocol is being developed. An evaluation protocol is necessary to determine how well they may perform in emergency response (real-time) situations. The evaluation of high-impact software must be conducted in accordance with WSRC QA Manual, 1Q, QAP 20-1.

This report will describe the method that will be used to evaluate the atmospheric models. The evaluation will determine the effectiveness of the atmospheric models in emergency response situations, which is not necessarily the same procedure used for research purposes. The format of the evaluation plan will provide guidance for the evaluation of atmospheric models that may be added to the WIND system in the future. The format of the final report will be similar to the procedure suggested in this document, except that detailed results of the evaluation will replace the description of the proposed plan.

The evaluation plan will consist of three parts, each designed to provide the user with information about the WIND system atmospheric models that is necessary for emergency response situations. The three parts are:

(1) Description: A brief description of the objectives, meteorological and source input parameters, model assumptions, and numerical procedures used in each of the atmospheric models will be included to provide background material for the evaluation. Other docurnentation that contains the detailed description of the model formulation will be referenced.

Evaluation: The goal of this evaluation is to make certain the atmospheric codes perform the specified theoretical calculations.

Documentation: Documentation will be written to record the results of the evaluation and to satisfy some of the required content of the software quality assurance test plan as listed in the WSRC QA Manual, 1Q, QAP 20-1.

The SQA (software quality assurance) test plans for the WIND system atmospheric models will reference the final evaluation document. 


\subsection{WIND SYSTEM ATMOSPHERIC MODELS}

The components of the WIND system atmospheric models to be evaluated will include AREA EVACUATION, PUFF/PLUME and 2DPUF. These models vary in complexity depending on the specific purpose they were designed for; therefore, different evaluation techniques will be required for each model. This section will describe the specific objective of each atmospheric model.

The WIND system atmospheric models can be accessed by SRS users through the Weather Center Analysis Laboratory (WCAL) computer system. The WCAL computer system consists of two Digital Equipment Corporation (DEC) microcomputers: currently two VAX 8550's clustered to provide disk shadowing for redundancy. A complete list of the WIND system computer terminals can be found in the WIND System User's Manual, WSRC TM-90-14.

\subsection{AREA EVACUATION Code}

The AREA EVACUATION code is a simple, straight-line Gaussian model because it is designed to predict the movement of potential releases close to the reactor areas; therefore, quick computations are necessary. Meteorological observations are used to calculate the plume direction, plume width, and dose estimates in the immediate vicinity of the release. The code uses the most recent wind data at a specific site and does not allow it to vary in space or in time.

\subsection{PUFF/PLUME Code}

The PUFF/PLUME code (Garrett and Murphy, 1981) is a modified Gaussian model designed to predict plume characteristics and perform dosimetry calculations for the site boundary and beyond due to a release at the site. The code uses the most recent wind data at the release site and allows it to vary in time according to a Model Output Statistics (MOS) forecast. MOS forecasts employ regression equations to link the observed tower data with predictions from the National Weather Service (NWS) Limited Fine Mesh (LFM) model.

\subsection{DPUF Code}

The 2.DPUF code (Addis and O'Steen, 1990) is a modified Gaussian model designed to predict more complex concentration distributions on and off-site to obtain accurate dosimetry calculations. NWS hourly surface observations and available upper-air observations throughout the southeast can be used to generate a regional wind field that varies horizontally, but is constant in time. NWS prediction models and MOS can also be used to calculate a time-dependent wind field that does not vary in space.

\subsection{DESCRIPTION OF WIND SYSTEM ATMOSPHERIC MODELS}

The WIND system atmospheric models have been used at the SRS for emergency response purposes for several years. Although the basic Gaussian prediction portion of the codes have changed little in recent years, these models have undergone extensive modifications due to upgrades in computer hardware and improved graphical capabilities. A description of these models is necessary to assess the accuracy of the mathematical framework, realism of the physical processes, and appropriateness of the assumptions. This description explains the need for a suite of models that independently represent the best solution to a given requirement or situation. The ability to select a model that provides the best prediction in a wide variety of possibilities is vital to emergency response applications. 


\subsection{Representation of the Physical Processes}

Straight-line Gaussian models suffer from limitations including the gross simplification of the atmospheric properties, the inability to represent recirculation of pollutants in complex terrain, and the erroneous use of one stability class at a given time to represent turbulent mixing over heterogeneous terrain. In this section, the assumptions of the AREA EVACUATION, PUFF/PLUME, and 2DPUF codes will be listed and the suitability of these codes to simulate dispersion in the SRS area will be discussed. All of the current atmospheric models in the WIND system are Gaussian in nature; however, PUFF/PLUME and 2DPUF have become more flexible because the underlying assumptions have been modified.

The assumptions of the WIND atmospheric models will also be compared to other similar Gaussian models. One of these models will include a regulatory model used by the Environmental Protection Agency (EPA).

\subsection{Numerical Procedure}

This section will include a general description of the numerical procedure used by the models to obtain a dispersion forecast. Much of this information has already been documented and will be referenced here as well. Flow charts are one method of depicting the overall numerical structure of a program. They have been used in Garrett and Murphy (1981) and Addis and O'Steen (1990) to depict the overall structure of the codes; however, they must be kept current to document the development of new hardware and graphical routines.

\subsection{Meteorological Input}

Meteorological towers and the TV tower in the SRS area collect wind speed, wind direction and turbulence data, along with temperature and dew-point temperature, that is used as input to the WIND system. Additional data used in certain WIND system atmospheric models comes from the National Weather Service (NWS) offices in Augusta and Washington D. C. This section will include a general description of the meteorological data that are used. The available temporal and spatial resolution of the data will also be documented and related to the atmospheric models.

The quality of this data base and the accuracy of the instruments on the SRS meteorological towers needs to be described because parameters such as wind speed, wind direction, horizontal standard deviation of the wind, $\sigma_{\mathrm{a}}$, and vertical standard deviations of the wind, $\sigma_{e}$, are employed by the dispersion models. Small errors in the wind direction and speed can lead to large errors in dispersion predictions over relatively large distances and times.

Much of this information, such as the on-site and off-site tower data, has already been documented and will be referenced here for convenience. The information on the field calibration procedures for the meteorological instruments (Procedure Manual L15.3), the quality assurance procedure for the meteorological data (Pendergast, 1980), and the reliability of the meteorological data will also be referenced.

\subsection{Source Input}

Gaussian models require instantaneous or continuous source data on chemical or radioactive materials. This section will describe how source data is obtained for the WIND system atmospheric models. During emergency response situations there may be a period of time when no source release data is known, but the atmospheric models need to be executed. When this occurs, default values for the source term are used in the models. These default values need to be described and examined. 


\subsection{WIND SYSTEM EVALUATION}

The WIND system atmospheric models have been shown to qualitatively predict the regions where toxic or radioactive material may be transported. This has been confirmed by comparing a number of tracer experiments performed at the site (Weber, 1984) to the results predicted by PUFF/PLUME and 2DPUF (Addis, 1985); however, no formal evaluation of the numerical codes of the atmospheric models has been performed.

The evaluation of the atmospheric models is done to test the logic and assumptions employed by the model code itself. Evaluation in this study refers to the process of examining and appraising the performance by comparing the model's concentration estimates to measurements. This evaluation will not be a verification procedure (prove the accuracy of a model) or a validation procedure (leads to formal recognition of a model) (Fox, 1981). During the evaluation, possible sources of error in the model results including input errors (due to meteorological and source input) and model errors (due to model assumptions and numerical procedures) will be identified.

One basis for acceptance of a new dispersion model involves the comparison of model results with those obtained from an established reference model. The new model would be accepted if if performed as well, or better, than the reference model. Another basis for accepting a new dispersion model could involve the use of performance criteria developed specifically for the intended application. Performance criteria should ensure that the model is technically sound and applicable to the problem. The evaluation of the WIND atmospheric models will incorporate both of these methods to determine whether the they can simulate the dispersion of toxic or radioactive material at the SRS site during emergency response situations.

Before an evaluation of a emergency response code can be made, the most relevant information must be determined. Obviously, an accurate prediction of the location and arrival time of toxic particles at a specific location is needed during an emergency response situation so that dose assessments can be made. Therefore, it is important to compare certain dispersion characteristics of the observed data with those predicted by the WIND atmospheric models. These characteristics should include (in order of importance for emergency response situations):

(1) Centerline location of plume or puff,

(2) Centerline concentration (peak and average),

(3) Width of plume or puff (peak and average),

(4) Overall integrated concentration in time at a particular location,

(5) Peak concentration estimates (uncorrelated in space and time),

(6) Peak concentration estimates (correlated spatially or temporally),

(7) Concentration estimates (correlated spatially or temporally).

The WIND system evaluation performance criteria may include:

(1) Comparing the model predictions with analytic solutions,

(2) Comparing the model predictions with observational data,

(3) Comparing the model predictions with those obtained from other dispersion models,

(4) Examining the sensitivity of the model predictions to variations in the input parameters,

(5) Examining the operational characteristics of the system.

The evaluation of each atmospheric model may not incorporate all of these performance criteria because the complexity of each model is different. The accuracy of the mathematical framework, realism of the physical processes, and appropriateness of assumptions can be determined by using this criteria. Each performance criteria may involve graphically displaying the observational data and numerical predictions of the concentrations, calculating and displaying statistics on the model 
performance, and segregating the observational data and numerical predictions into different stability regimes in which dispersion processes are understood.

This is a general guideline which may have to be modified when additional atmospheric models are added to the WIND system in the future.

\subsection{Comparison Against Analytic Solutions}

Analytic solutions of the Gaussian equations used by the WIND system atmospheric models exist for both the instantaneous and continuous point sources assuming simple meteorological conditions. The atmospheric models can be executed with the same simple meteorological conditions so that the numerical results may be compared to the analytic solutions. The objective of this method is to evaluate some of the logic in the code. Large differences may indicate an error or an incorrect assumption in the model code. Small differences could be attributed to truncation errors and would indicate the model is predicting the theoretical dispersion characteristics correctly.

Any atmospheric model on the WIND system which can have the governing equations reduced sufficiently so that an analytic solution exists should be evaluated in this manner.

A general procedure for the evaluation of a numerical code could be:

(1) Choose the default input parameters in the model that would satisfy the Gaussian solution for a continuous or instantaneous source. The default input parameters could include wind direction, $\mathrm{q}$, wind speed, $\mathrm{V}$, horizontal standard deviation of the wind, $\sigma_{\mathrm{a}}$, vertical standara deviation of the wind $\sigma_{e}$, source strength, $Q$, and specific chemical or radionuclide.

Create a file of the predicted dispersion pattern as computed by the code. The format of the file should list the coordinates, the time of arrival, the concentration, the computed horizontal standard deviation of the wind, $\sigma_{x}$, and the vertical standard deviation of the wind $\sigma_{z}$ at each location downwind of the source at the ground centerline. This file may also list information in the vertical direction along the centerline. Horizontal distributions perpendicular to the centerline can be inferred from the standard deviations.

(3) Create a file of the dispersion pattern of the analytic Gaussian solution made by an independent calculation with the same input parameters as the code.

(4) Calculate the difference between the results of the code and the analytic Gaussian solution.

(5) Modify one input parameter in both the code and the analytic Gaussian solution. Repeat steps (2) to (4).

(6) Repeat steps (2) to (5) until the desired number of input parameters are changed in the model evaluation.

The purpose in modifying the default input parameters is to verify that the plume directions are opposite to wind directions, arrival times at each downwind location will decrease (increase) with increasing (decreasing) wind speed, lateral spread of pollutants will increase (decrease) with increasing (decreasing) horizontal variation in the winds, the vertical spread of pollutants will increase (decrease) with increasing (decreasing) vertical variation in the winds, and concentrations will increase (decrease) with increasing (decreasing) source strength. The tests against the analytic solutions can also be used to verify certain limiting cases of diffusion, such as the direction of the plume centerline, the independence of concentration with height far from the source. Other experiments may be performed to test the sensitivity of the simulated diffusion to source height and the high/low wind speeds.

The total number of simulations to be performed for the parameter variations listed above will depend on the specific atmospheric model. The Gaussian solution would have to be calculated for 
the same parameter variations. Some modification to the codes will have to be made to print out all the required variables.

\subsection{Comparison Against Observational Data}

Analytic solutions are useful to evaluate the atmospheric models for simple meteorological situations; however, observational data are necessary to assess the rnodel behavior in more complicated, realistic flow fields. The objective of this method is to test a greater portion of the code using observed meteorological data and data from tracer studies. Some preliminary evaluation of the PUFF/PLUME code with the MATS data (Smith, 1984; Addis, 1985) has been performed; however, additional studies must be done for the current versions of the atmospheric models in the WIND system to test their individual responses.

The model performance can be appraised by using real meteorological data and observational tracer studies such as Prairegrass (Lettau and Davidson, 1957), MATS (Weber, 1984), and STABLE (Weber and Kurzeja 1991). The specific subsets of data from these experiments to be used in the evaluations will depend upon the particular atmospheric model. The atmospheric models may also be executed to simulate observed dispersion releases performed at locations other than at the SRS.

The general procedure for this portion of the model evaluation will be:

(1) Execute the model with the meteorological conditions from a single tracer study,

(2) Graphically display model results and observed fields,

(3) Apply statistics to model results,

(4) Repeat steps (1) to (3) for a number of experiments (the exact number to be determined later),

(5) Compute statistics for entire series of experiments used in steps (1) to (4). This procedure may involve segregating the data into different stability regimes,

Since the MATS and STABLE data sets are based on a tracer chemical release, the deposition, radionuclide, and radioactive decay portions of the WIND atmospheric models cannot be evaluated. Other methods of evaluation for these portions of the code will have to be determined. The Tritium gas oxide data from offsite samplers and the Krypton measurements near the SRS (Heffter, 1984; Pendergast et al., 1979) may be used to evaluate other portions of the corle.

Graphical display of data is necessary to get an intuitive feel for the model results as compared with the observations. Statistics alone may be deceiving and it is always advisable to examine both the raw observational and numerical data.

Statistics are necessary to quantitatively evaluate the important characteristics of dispersion for emergency response including the centerline location, width of plume or puff, overall integrated concentration, and peak concentration estimates. The evaluation of the WIND system models may employ the following statistical parameters to quantitatively compare model results and observations:

(1) absolute difference, $d$,

(2) variance, $s^{2}$,

(3) mean-square error, MSE,

(4) correlation, $r$,

(5) ratio methods, $R$,

(6) statistical tests, $t$ and $F$. 
Fox (1981), Hanna (1984), and Dickerson and Ermak (1988) have suggested that these statistics can be used to evaluate dispersion models. There have been many published air-quality studies that employ these statistics, such as those reported in the Hilton Head (Buckner, 1980) and Kiawah (Weber and Garrett, 1984) workshops. As described in Dickerson and Ermak (1988), there are inherent protlems with the difference and ratio methods; however, they can be modified so that meaningful results can be obtained. Additional parameters should be calculated, such as the integrated concentration with time, to get a more accurate overall dose assessment. Each of these statistics may not be computed for every model run; however, the model and observational data sets should be extensive enough so that these quantities could be computed if necessary. It is recommended that similar statistics be used for the evaluation of each of the WIND atmospheric models.

As in the analytic evaluation section, additional information on the concentration values can be printed out to verify overall model behavior. This would include computing the vertical and crosswind concentrations in addition to the ground concentrations. This is optional information, that may not be necessary, since these parameters are not critical forecast variables for emergency response purposes.

\subsection{Comparison Against Other Atmospheric Models}

The numerical results of a particular WIND system atmospheric code should be compared to those obtained from other atmospheric/dispersion models that can be applied to the SRS area. Three types of models to be used in this comparison include:

(1) Other models on the WIND system,

(2) Off-site models,

(3) EPA models.

The predictions made by one particular code could be compared with those made by simpler codes in the WIND system. This can be done either to verify that two models may produce nearly identical results given certain assumptions and simplifications, or to verify that one model can more accurately simulate dispersion from an accidental release given the default parameters. For instance, the results of 2DPUF could be comf red with those from PUFF/PLUME, and the results from PUFF/PLUME could be compared witr. nose from AREA EVACUATION.

The numerical results of the atmospheric models in the WIND system should be compared with other published models. There have been a number of studies that have used the MATS data set to evaluate other numerical dispersion models. Weber and Garrett (1984) described the results of several models that used the MATS data set presenter at the Kiawah workshop. These models ranged in complexity from simple Gaussian models to three-dimensional, time-dependent models of the atmosphere, such as the Atmospheric Diffusion Particle-In-Cell (ADPIC) model (Rodriquez and Rosen, 1984). The results of the observational evaluation by the WIND system model could be modified to present the same type of output as those presented at the Kiawah workshop. The numerical results of the atmospheric models may also be compared with other dispersion studies performed at locations other than at the SRS.

The results of an EPA model using the MATS data set were also presented at the Kiawah workshop (Weber, 1984). The results of the WIND system evaluation can be used to compare with these studies. An EPA regulatory model can be employed to simulate the dispersion in the MATS or STABLE experiments. The results from this model would then be compared statistically and qualitatively to the observations and the predictions made by the WIND atmospheric models. 


\subsection{Sensitivity Analysis}

The sensitivity of the model results to certain parameters in the model should also be examined to determine which parameters are critical for a dispersion forecast. In this way, some of the errors in the model results can be attributed to uncertainties in the meteorological data or to the model numerics itself.

One way to evaluate the atmospheric model's sensitivity to perturbations to model input is to use the Latin Hypercube Sample (LHS) method. The LHS experiment would proceed as follows:

(1) Determine which parameters are important in the atmospheric models,

(2) Determine the range of each parameter,

(3) Determine the distribution of the values of each of the parameters (uniform, normal, logirithmic, etc.),

(4) Ruı a Latin hypercube program to obtain random values of input parameters,

(5) Run each of the atmospheric models with the input parameters generated by the Latin hypercube program,

(6) Generate graphical results of the statistics from the output of the atmospheric models.

The results of the LHS experiments should indicate how sensitive the atmospheric models are to certain input parameters.

\subsection{Operational Characteristics}

Since the atmospheric models are to be used for emergency response simulations, speed of the computations is of utmost importance. A table of the current execution times on the VAX 8550 computer for each of the atmospheric models will be made. This table would also include model executions with limited amount of input (canned data) and executions that are strictly interactive. The amount of computer time spent in accessing input data, performing transport calculations, and producing graphical output of the results should be examined.

The reliability of the model and the computer for use during emergency response has been determined and will be documented here as well. The availability of the meteorological data for the atmospheric models will also be discussed.

There are other characteristics of the WIND system atmospheric models that may be important for emergency response situations that will have to be examined. For instance, the coding of the models does not permit a mistake on the part of the user. If a mistake is made, the model run must be aborted and restarted. This may waste valuable time in real emergencies. Testing should also be sufficient to ensure that the model does not perform an unintended function and degrade the results when invalid input information is used.

\subsection{CONCLUSION}

The product of the evaluation will be a summary of the tests performed on the WIND system atmospheric atmospheric models. Some of the tests will be required for the evaluation process, while other tests may be optional and more useful for research purposes. The results from the required tests of the evaluation should indicate whether they are adequate to simulate the dispersion of toxic materials at SRS in emergency response situations. A general evaluation plan, as presented in this document, should be agreed upon by the appropriate personnel in the ETG (Environmental Transport Group) of the ETS (Environmental Technology Section).

The acceptance of the atmospheric models will be based on the scientific judgment of the owners of the atmospheric codes (in accordance with ETS SQA, 1Q31-4) as well as a peer review by certain 
ETG personnel. The acceptance criteria would be based on information presented in sections 3 and 4. It is recommend that the following tasks be used for the WIND system atmospheric models (in order of importance):

\section{AREA EVACUATION:}

(1) Comparison Against Analytic Solutions (as listed in section 4.1, for plume),

(2) Comparison Against Other Atmospheric Models (as listed in section 4.3, using an EPA model),

(3) Operational Characteristics (as listed in section 4.5, do as much as possible),

(4) Comparison Against Observational Data (as listed in section 4.2, using a small data set),

\section{PUFF/PLUME:}

(1) Comparison Against Analytic Solutions (as listed in section 4.1, for a puff and a plume),

(2) Comparison Against Observational Data (as listed in section 4.2, using extensive MATS and STABLE data sets),

(3) Comparison Against Other Atmospheric Models (as listed in section 4.3, using the AREA EVACUATION and an EPA model, referencing other models),

(4) Operational Characteristics (as listed in section 4.5, do as much as possible),

2DPUF:

(1) Comparison Against Analytic Solutions (as listed in section 4.1, for a puff),

(2) Comparison Against Observational Data (as listed in section 4.2, with extensive MAT'S and STABLE data sets),

(3) Comparison Against Other Atmospheric Models (as listed in section 4.3, using the PUFF/PLUME and an EPA model, referencing other models),

(4) Operational Characteristics (as listed in section 4.5, do as much as possible).

The work performed for this project is expected to involve the contributions of several individuals to expedite the evaluation process including:

(1) The WIND system evaluation coordinator

(2) The custodians of the atmospheric models

(3) Other scientists in the ETS

The WIND system evaluation coordinator will write the description of the atmospheric models, perform all of the statistics in this study, disseminate and display the data, and describe the results. The custodians of the atmospheric models are expected to help modify the numerical codes so that the appropriate variables can be computed and tabulated for the analytic, observational, and LHS evaluation studies. Other ETS scientists will be consulted for assistance in performing miscellaneous tasks for this project. Assistance will be needed in accessing and compiling the appropriate observational data from the MATS and STABLE experiments, solving computational problems, obtaining information on the LHS program, and obtaining information on an EPA regulatory model.

Major portions of the evaluation are scheduled for completion by September 30, 1991. 


\subsection{REFERENCES}

Addis, R. P., 1985, "An Evaluation of SRL Emergency Response Codes using MATS Data", DPST-850715. DuPont de Nemours and Company, Savannah River Laboratory, Aiken, $\mathrm{SC}$.

Addis, R. P, and L. O'Steen, 1990, "2DPUF, A Sequential Gaussian Puff Model", WSRC-RP90-1208. Westinghouse Savannah River Company, Savannah River Laboratory, Aiken, $\mathrm{SC}$.

Buckner, M. R., 1980, Proceedings of the First SRL Model Validation Workshop. Kiawah Island, South Carolina, November 19-21, DP-1597 DuPont de Nemours and Company, Savannah River Laboratory, Aiken, SC.

Dickerson, M. H., and D. L. Ermak, 1988, "The Evaluation of Emergency Response Trace Gas and Dense Gas Dispersion Models", UCRL,-99348, Lawrence Livermore National Laboratory, Lawrence, CA.

Fox, D. G., 1981, "Judging Air Quality Model Performance", Bull. Amer. Meteor. Soc., 62:599609.

Garrett, A. J., and C. E. Murphy Jr., 1981, "A Puff-Plume Atmospheric Deposition Model for use at SRP in Emergency Response Situations", DP-1595. DuPont de Nemours and Company, Savannah River Laboratory, Aiken, SC.

Hanna, S. R., 1984, "A Simple Method of Evaluating Air Quality Models", Proceedings of the DOE/AMS air pollution model evaluation workshop. Kiawah Island, South Carolina, October 23-26, 1984, Vol. 1. DP-1701-1. DuPont de Nemours and Company, Savannah River Laboratory, Aiken, SC.

Heffter, J. L., 1984, "Atlantic Coast Unique Regional Atmospheric Tracer Experiment (ACURATE)", NOAA Techincal Memoradum ERL ARL-130, Air Resources Laboratory, Rockville, MD, $15 \mathrm{pp}$.

Lettau, H. H., and B. Davidson, 1957, "Exploring the Atmosphere's First Mile", Vol. I., Instrumentation and Data Evaluation, Vol. 2, Site Description and Data Tabulation. Pergamon Press.

Pendergast, M. M., 1980, "Real Time Quality Control of Meteorological Data Used in SRP's Emergency Response System", DP-1551. DuPont de Nemours and Company, Savannah River Laboratory, Aiken, SC.

Perdergast, M. M., A. L. Boni, G. J. Ferber, and K. Telgadas, 1979, "Measured Weekly Kr-85 Concetrations Within $150 \mathrm{~km}$ of the Savannah River Plant (March $1975 \mathrm{~T}$.rough August 1976), DP-1486. DuPont de Nemours and Company, Savannah River Laboratory, Aiken, $\mathrm{SC} ., 56 \mathrm{pp}$.

Rodriquez, D. J,, and L. C. Rosen, 1984, "The Evaluation of a Series of SF-6 Tracer Releases using the MATHEW/ADPIC Model", Proceedings of the DOE/AMS air pollution model evaluation workshop. Kiawah Island, South Carolina, October 23-26, 1984, Vol. 1. DP1701-1. DuPont de Nemours and Company, Savannah River Laboratory, Aiken, SC. 
Smith, F. G., 1984, "Evaluation of a Gaussian Plume Model using MATS Data", Proceedings of the DOE/AMS air pollution model evaluation workshop. Kiawah Island, South Carolina, October 23-26, 1984, Vol. 1. DP-1701-1. DuPont de Nemours and Company, Savannah River Laboratory, Aiken, SC.

Weber, A. H., 1984, "The MATS (Mesoscale Atmospheric Transport Studies) Experiments", Proceedings of the DOE/AMS air pollution model evaluation workshop. Kiawah Island, South Carolina, October 23-26, 1984, Vol. 1. DP-1701-1. DuPont de Nemours and Company, Savannah River Laboratory, Aiken, SC.

Weber, A. H., and A. J. Garrett, 1984, "Proceedings of the DOE/AMS Air Pollution Model Evaluation Workshop", Kiawah Island, South Carolina, October 23-26, Vol. 1 DP-12017, DuPont de Nemours and Company, Savannah River Laboratory, Aiken, SC.

Weber, A. H., and R. J. Kurzeja, 1991, "Nocturnal Planetary Boundary Layer Structure and Turbulence Episodes During the Project STABLE Field Program", (to appear in J. Applied Meteor.) 
:
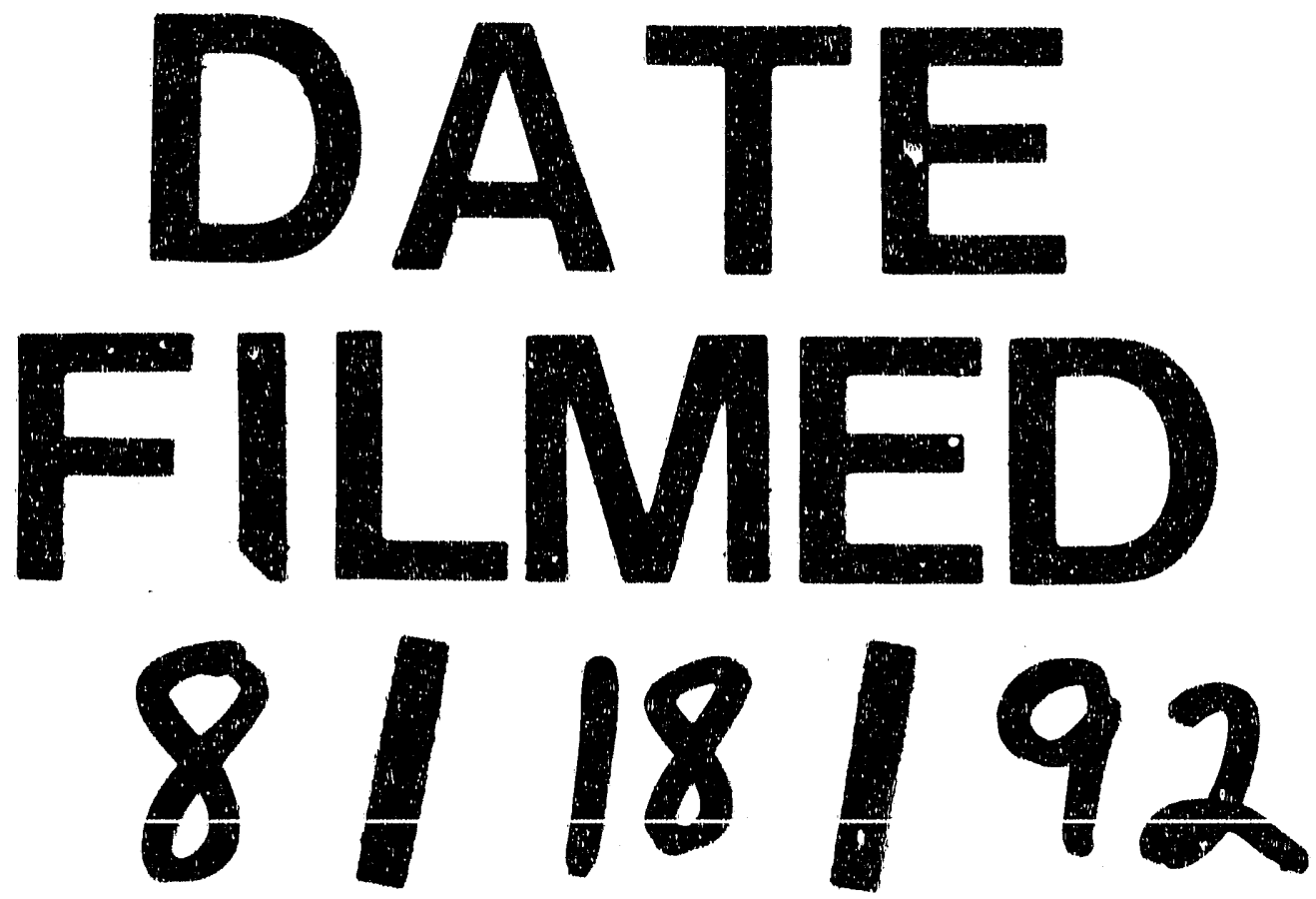
I 